\title{
Autopercepção da saúde entre usuários da Atenção Primária em Porto Alegre, RS
}

\author{
Self-perceived health of adults users of Primary Health Care Services in \\ Porto Alegre, RS, Brazil
}

Milena Rodrigues Agostinho', Mônica Celestina Oliveira², Maria Eugênia Bresolin Pinto³, Giuliano Uhlein Balardin ${ }^{4}$, Erno Harzheim ${ }^{5}$

Palavras-chave: Atenção Primária à Saúde

Autoimagem

Estudo transversais

\section{Resumo}

A autopercepção da saúde baseia-se em critérios subjetivos e objetivos, sendo influenciada por fatores como sexo, idade, classe social e presença de doenças crônicas. Este trabalho descreve a autopercepção da saúde de usuários adultos adscritos à rede de Atenção Primária à Saúde (APS) de Porto Alegre (RS). Trata-se de um estudo transversal de base populacional, que avaliou a autopercepção da saúde por meio da pergunta "Comparado com alguém de sua idade e sexo, como você considera sua saúde?". As respostas foram agrupadas em "boa" ou "ruim". Variáveis sociodemográficas e econômicas, biológicas, de estilo de vida, do estado de saúde e da relação com o Serviço de Saúde ou médico referido foram utilizadas no modelo hierárquico conceitual para auxiliar na descrição da autopercepção de saúde por meio de regressão de Poisson modificada. De 3.009 usuários, 2.355 (78,3\%; IC: 74,9-81,3) referiram ter boa autopercepção de saúde, dos quais 1.013 (43\%; IC: 39,3-46,9) eram homens, 1.229 pertenciam às classes sociais C, D e E (52,2\%; IC: 40,2-63,9), 629 (26,7\%; IC: 26,5-30,1) pertenciam à faixa etária de 18 a 30 anos e 626 (26,6\%; IC: 21,5-31,5) à faixa etária de 30 a 45 anos. Dos que referiram boa autopercepção de saúde, 1.075 eram tabagistas (45,7\%; IC: 40,9-50,6), 276 consumiam mais de 175g de álcool/semana (11,7\%; IC: 10,2-13,4), 1.197 eram sedentários (51,4\%; IC: 49,1-53,7) e 573 possuíam pelo menos uma doença crônica (24,4\%; IC: 20,9-28,2). Pelo modelo hierárquico, observa-se que sexo feminino (RP: 0,92; IC: 0,88-0,95), tabagismo (RP: 0,92; IC: 0,88-0,96), presença de doença crônica (RP: 0,78; IC: 0,72-0,84) e utilização do Serviço - pelo menos quatro consultas/ano (RP: 0,85; IC: 0,82-0,92) foram fatores que diminuem a probabilidade de uma pessoa referir boa autopercepção de saúde. Não ter hospitalização (RP: 1,14; IC: 1,06-1,25) e estar satisfeito com a última consulta (RP: 1,19; IC: 1,11-1,27) aumentaram significativamente a probabilidade de 0 usuário avaliar sua saúde como boa. A autopercepção da saúde mostra-se influenciada por fatores biológicos, socioeconômicos e de vínculo com o Serviço. Cabe ressaltar que maior satisfação com Serviço de APS estava associada à melhor autopercepção de saúde, justificando a reorganização do Sistema Único de Saúde pela ampliação da rede de Serviços de APS, como a Estratégia Saúde da Família.

\footnotetext{
${ }^{1}$ Acadêmica do Curso de Medicina da Universidade Federal do Rio Grande do Sul (UFRGS); Bolsista do Grupo de Pesquisa em Atenção Primária à Saúde de Porto Alegre, Programa de Pós-Graduação em Epidemiologia da UFRGS, Porto Alegre (RS), Brasil.

${ }^{2}$ Estatística; Doutoranda do Programa de Pós-Graduação em Epidemiologia da UFRGS; Pesquisadora do Grupo de Pesquisa em Atenção Primária à Saúde de Porto Alegre, Programa de Pós-Graduação em Epidemiologia da UFRGS, Porto Alegre (RS), Brasil.

${ }^{3}$ Doutoranda do Programa de Pós-graduação em Epidemiologia da UFRGS; Médica de Família e Comunidade; Professora Assistente do Departamento de Saúde Coletiva da Fundação Universidade Federal de Ciências da Saúde de Porto Alegre (UFCSPA); Pesquisadora do Grupo de Pesquisa em Atenção Primária à Saúde de Porto Alegre, Programa de Pós-Graduação em Epidemiologia da UFRGS, Porto Alegre (RS), Brasil.

${ }^{4}$ Psicólogo; Integrante do Grupo de Pesquisa em Atenção Primária à Saúde de Porto Alegre, Programa de Pós-Graduação em Epidemiologia da UFRGS, Porto Alegre (RS), Brasil.

${ }^{5}$ Doutor em Medicina Preventiva e Saúde Pública pela Universidade de Alicante, Espanha; Médico de Família e Comunidade; Professor Adjunto do Departamento de Medicina Social da Faculdade de Medicina da UFRGS e do Programa de Pós-Graduação em Epidemiologia da UFRGS; Líder do Grupo de Pesquisa em Atenção Primária à Saúde de Porto Alegre, Programa de Pós-Graduação em Epidemiologia da UFRGS; Líder do Grupo de Pesquisa
da UFRGS, Porto Alegre (RS), Brasil.

Endereço para contato: Milena Rodrigues Agostinho, Rua Coronel João Correa, 110, apto. 105 - Passo da Areia, Cep: $91350-190$
} Porto Alegre/RS, Telefone: (51) 9293-7559, E-mail: milena.rodrigues.agostinho@gmail.com 


\begin{abstract}
Key Words: Abstract
Primary Health Care

Self Concept

Cross-sectional Studies

Self-perceived health is based on both subjective and objective criteria and can be influenced by factors such as gender, age, social class and existing chronic disease. This paper describes self-perceived health in adults affiliated to the network of Primary Health Care (PHC) in the city of Porto Alegre (RS), Brazil. This is a population-based cross-sectional study. Self-perceived health was assessed based on the question: "Compared to someone from your age and gender, how would you rate your health?". Responses were assembled as "good" or "bad". Socioeconomic and demographic variables, as well as biological effects, lifestyle, health status and the relationship between patients and the Health Care System or doctors have been used in the hierarchical conceptual model in order to help the description of self-perception of health through modified Poisson regression. From 3,009 users, 2,355 (78.3\%; Cl: 74.9-81.3) believed to have good self-perceived health. Out of the total 1,013 (43\%; Cl: 39.3-46.9) were men, 1,229 belonged to C, D and E social classes (52.2\%; Cl: 40.2-63.9), 629 (26.7 \%; IC: 26.5-30.1) belonged to age 18 to 30 years and 626 (26.6\%; IC: 21.5-31.5) to 30 to 45 years of age. From those having self-perceived their health as "good", 1,075 smoked (45.7\%; Cl: 40.9-50.6), 276 consumed more than $175 \mathrm{~g}$ of alcohol per week (11.7\%; Cl: 10.2-13.4), 1,197 were sedentary (51.4\%; Cl: 49.1-53.7) and 573 suffered at least from one chronic disease (24.4\%; Cl: 20.9-28.2). By means of hierarchical model, we have observed that the probability of a person referring to his/her self-perceived health as good, diminished if they were women (PR: 0.92; Cl: 0.88-0.95), smokers (PR: 0.92; Cl: 0.88-0.96), had a chronic disease (PR: 0.78; Cl: 0.72-0.84), or were users of the Health Services - at least four appointments per year (PR: 0.85; Cl: 0.82-0.92). Conversely, not having been hospitalized (PR: 1.14; Cl: 1.06-1.25) or been satisfied with the latest appointment (PR: 1.19; $\mathrm{Cl}: 1.11-1.27)$ increased significantly the probability of a user to assess its health as "good". Self-perceived health has proved to be influenced by biological and socialeconomic factors as well as existing attachment to health services. It should be noted that the more satisfied a patient was with PHC services, the better self-perception of health he had, which justifies the reorganization of the Brazilian national health care service (Sistema Único de Saúde) through the extension of present PHC network services, such as the Family Health Strategy (Estratégia Saúde da Família) program.
\end{abstract}

\section{Introdução}

A Atenção Primária à Saúde (APS) constitui-se como a porta de entrada do sistema de saúde. Suas principais características são: acesso de primeiro contato, a longitudinalidade, integralidade e coordenação do cuidado, além da orientação familiar e comunitária e da competência cultural ${ }^{1}$. A reorganização do Sistema Único de Saúde (SUS), com o fortalecimento da APS por meio da Estratégia Saúde da Família $(\mathrm{ESF})^{2}$, está auxiliando na diminuição das iniquidades no acesso aos Serviços de Saúde ${ }^{3}$ e também na saúde da população em geral ${ }^{4-6}$. Avaliar a saúde da população adscrita às unidades de Atenção Primária é condição fundamental para o reconhecimento do território de ação dessas equipes. Entre os indicadores recomendados pela Organização Mundial de Saúde (OMS) para esse processo estão a autopercepção do estado de saúde, a percepção de doença de longa duração e a qualidade de vida, ressaltando que os resultados desses indicadores podem ser comparados a outras populações e diferentes momentos no tempo ${ }^{7}$.

A autopercepção da saúde baseia-se em critérios subjetivos e objetivos, sendo um bom preditor de mortalidade e refletindo uma percepção individual, que inclui aspectos biológicos, psicológicos e sociais ${ }^{8}$. Ela tem influência de fatores como condições socioeconômicas, sexo, idade e presença de doenças crônicas ${ }^{9,10}$. É essencial entender como a pessoa percebe sua saúde, pois o seu comportamento é condicionado pela percepção e pela importância dada a esta ${ }^{11}$. Muitas vezes, portadores de doenças crônicas não se percebem doentes, principalmente por não apresentarem sintomas (por exemplo, hipertensão arterial sistêmica), não serem, ainda, portadores de incapacidades ou não apresentarem, em dado momento, outras comorbidades. Em estudo ${ }^{10}$ que avaliou a percepção de saúde, evidenciou-se que homens percebem menos sua saúde como ruim quando comparado às mulheres, o que afeta a utilização dos Serviços de Saúde por esses usuários.

A APS permite aos profissionais formarem um forte e contínuo vínculo com a população atendida (longitudinalidade), o que favorece a promoção de saúde, a prevenção de doenças e o diagnóstico precoce. Um dos recursos que favorece o desempenho dos profissionais de saúde é o conhecimento da comunidade em que o paciente habita, focando seu trabalho não na doença, mas nas pessoas e em seu ambiente físico, social e cultural (orientação comunitária). Desse modo, exploram-se as inter-relações entre as várias dimensões da saúde, obtendo uma percepção integrada do indivíduo ${ }^{10}$ (integralidade). A doença não se refere apenas à dor física e ao desconforto, mas também às consequências sociais e psicológicas de conviver com o processo de adoecimento.

O presente estudo teve por objetivo descrever a autopercepção da saúde de usuários adultos adscritos à rede de APS de Porto Alegre (RS), identificando os fatores associados à boa percepção de saúde.

\section{Métodos}

Foram utilizados os dados de um estudo transversal de base populacional realizado nos Serviços de APS do município de Porto Alegre ${ }^{12}$. Participaram deste estudo usuários adultos, 
maiores de 18 anos, residentes nas áreas geográficas cobertas pela rede pública de APS e de um Serviço privado. A amostragem foi realizada por conglomerados, sendo calculada para estudos transversais comparativos e para descrição de proporções, com amostra total igual a 3.009 usuários. A coleta dos dados foi realizada no período de agosto de 2006 a junho de 2007.

Entre as Unidades Básicas de Saúde (UBS) e a ESF, foram selecionados aleatoriamente $50 \%$ das equipes de cada gerência distrital. Todas as unidades de APS do Grupo Hospitalar Conceição (GHC), que conta com 12 unidades, do Centro de Saúde Escola Murialdo (CSEM), que tem 7 unidades, e da Caixa de Assistência dos Funcionários do Banco do Brasil (CASSI), que conta com 5 unidades, foram incluídas na avaliação. Com base na estimativa de adultos por domicílio, foram sorteados aleatoriamente setores censitários para cada unidade de serviço amostrado. Dentro do setor censitário amostrado, foram selecionados domicílios de forma sistemática, sendo entrevistados todos os moradores adultos que preencheram os critérios de inclusão. Na CASSI, a amostragem dos adultos ocorreu proporcionalmente ao número de usuários cadastrados nas equipes e também foi obtida por amostragem aleatória simples. Todos os adultos dessas famílias foram contatados para participar do estudo.

Os adultos que preenchiam os critérios de inclusão e aceitaram participar da pesquisa, após leitura e assinatura do termo de consentimento livre e esclarecido, foram entrevistados. Foram excluídos os indivíduos que não apresentaram condições físicas ou mentais para responder ao questionário, que realizaram sua última consulta em um Serviço de Saúde de Porto Alegre antes de janeiro de 1996 (antes da municipalização) ou que referiram um Serviço de Saúde fora de Porto Alegre. Maior detalhamento da metodologia utilizada pode ser livremente acessado no artigo de referência ${ }^{12}$.

Utilizou-se, para a coleta das informações, um questionário geral, estruturado e composto por três instrumentos distintos:

1. questionário sobre a qualidade da APS: Instrumento de Avaliação da Atenção Primária, na sua versão adulto (PCATool-Brasil, Adult Primary Care Assessment Tool), composto por blocos de perguntas que correspondem aos atributos da APS (acesso, longitudinalidade, integralidade, coordenação, orientação familiar e orientação comunitária) e por três perguntas iniciais que medem o grau de afiliação ao Serviço de Saúde. As respostas são do tipo Likert ${ }^{13}$;

2. questionário estruturado com variáveis sociodemográficas e sobre os fatores de risco cardiovasculares;

3. questionário de satisfação do usuário (validado no Brasil, composto por 12 perguntas sobre os distintos aspectos da atenção - acesso, cordialidade, confiança, atuação do médico, orientações, marcação de consultas, avaliação geral - e com respostas tipo Likert de 1-5, representadas por figuras de faces com cinco expressões distintas de satisfação, que variam de "muito ruim" a "muito boa") ${ }^{14}$.

A autopercepção da saúde foi avaliada por meio da pergunta "Comparado com alguém de sua idade e sexo, como você considera sua saúde?". As respostas foram agrupadas em duas categorias: boa autopercepção de saúde foi definida pelas respostas "muito boa" e "boa", e ruim, pelas respostas "regular", "ruim" e "muito ruim". O estrato social foi definido por meio do Critério Brasil 2008 da Associação Brasileira de Institutos de Pesquisa de Mercado (Abipeme). Para cor da pele, utilizou-se a cor referida pelo entrevistado, mesma estratégia utilizada pelo Instituto Brasileiro de Geografia e Estatística (IBGE). A comorbidade expressa a presença de pelo menos um dos desfechos crônicos avaliados (diabetes mellitus, hipertensão, cardiopatia isquêmica e obesidade). Escolaridade foi definida por número de anos completos de estudo. Foi estipulado um ponto de corte como prejudicial à saúde o consumo de álcool acima de $175 \mathrm{~g}$ por semana ${ }^{15}$. Moradia era considerada não-segura quando construída com papelão, lata ou madeira irregular.

As variáveis utilizadas no modelo hierárquico conceitual (Figura 1) para auxiliar na descrição da autopercepção de saúde foram: idade, sexo, cor da pele, estado marital, classe

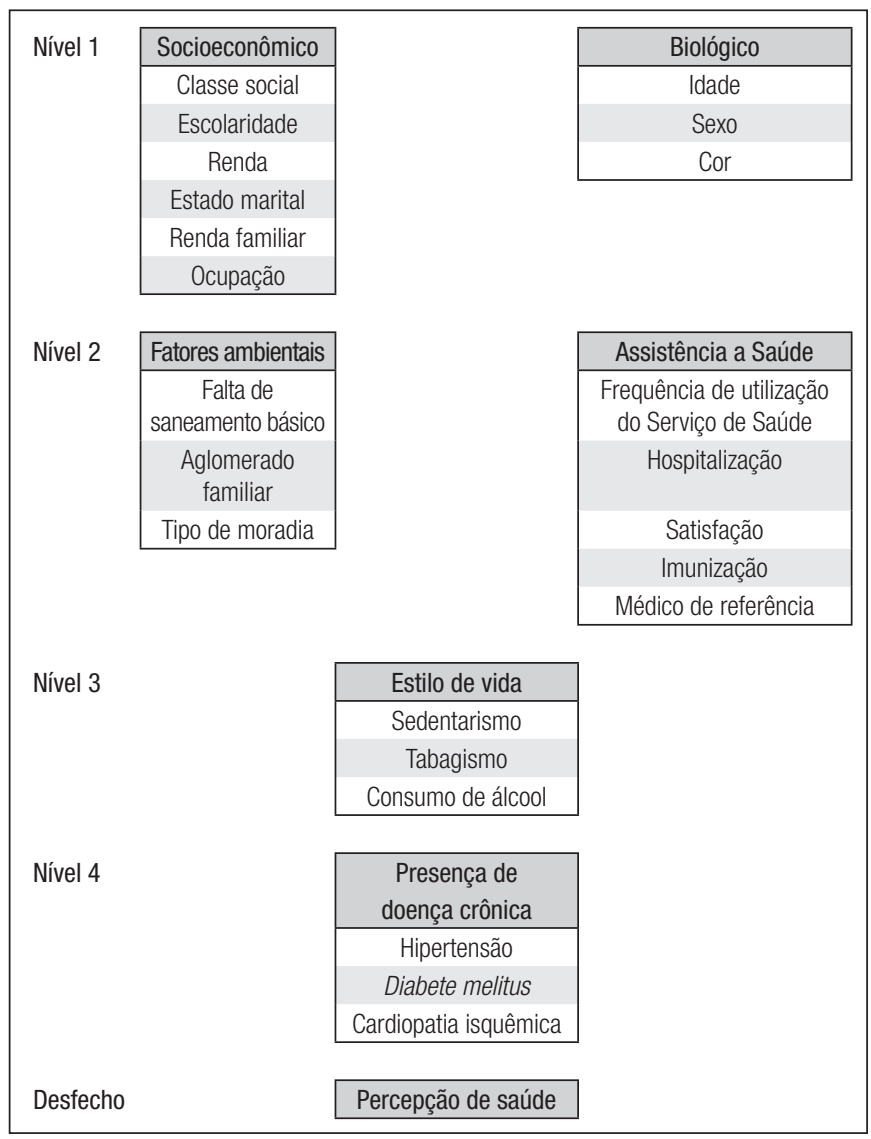

Figura 1: Modelo hierárquico conceitual. 
social, educação, renda, ocupação, saneamento básico, aglomerado familiar, tipo de moradia, imunização (vacina para gripe e pneumococo), tabagismo, consumo de álcool, sedentarismo, presença de doenças crônicas, hospitalizações, tipo de Serviço, médico de referência, frequência de utilização do serviço e satisfação. A associação das variáveis explicativas

Tabela 1: Características da população adscrita aos Serviços de Atenção Primária à Saúde, Porto Alegre, 2007

\begin{tabular}{|c|c|}
\hline Variáveis Sociodemográficas & Média (DP)/\%(IC) \\
\hline Idade (anos) & $45,8(17,3)$ \\
\hline \multicolumn{2}{|l|}{ Sexo } \\
\hline Masculino & $40,5(37,0-44,2)$ \\
\hline Feminino & $59,5(55,8-63,0)$ \\
\hline Escolaridade (anos completos) & $9,5(4,7)$ \\
\hline \multicolumn{2}{|l|}{ Cor da pele } \\
\hline Brancos & $68,5(61,4-74,8)$ \\
\hline Não brancos & $31,5(25,2-38,6)$ \\
\hline \multicolumn{2}{|l|}{ Classe Social } \\
\hline$A B$ & $44,7(33,5-56,5)$ \\
\hline CDE & $55,3(43,5-66,5)$ \\
\hline \multicolumn{2}{|l|}{ Estado marital } \\
\hline Casado & $61,1(59,3-62,9)$ \\
\hline Solteiro & $22,1(19,9-24,5)$ \\
\hline Viúvo/divorciado & $16,8(15,3-18,3)$ \\
\hline Renda per capita $(\mathrm{R} \$)$ & $956,2(1.286,5)$ \\
\hline Renda familiar (R\$) & $2776,7(3251,5)$ \\
\hline \multicolumn{2}{|l|}{ Ocupação } \\
\hline Empregado & $30,5(26,8-34,5)$ \\
\hline Autônomo/dono & $14,3(12,5-16,2)$ \\
\hline Com benefício & $25,2(20,0-31,1)$ \\
\hline Estudante/dona de casa & $22,9(21,0-24,9)$ \\
\hline Desempregado & $7,1(5,1-9,9)$ \\
\hline Saneamento básico & $98,2(97,2-98,8)$ \\
\hline Moradores por casa & $3,6(1,6)$ \\
\hline Moradia segura & $97,3(95,6-98,3)$ \\
\hline Estado de saúde & Média (DP)/\%(IC) \\
\hline Hipertensão arterial & $25,2(22,2-28,6)$ \\
\hline Diabetes mellitus & $7,1(5,8-8,6)$ \\
\hline Cardiopatia isquêmica & $5,8(4,6-7,4)$ \\
\hline Sedentarismo & $51,9(49,5-54,3)$ \\
\hline Tabagismo & $47,6(43,1-52,2)$ \\
\hline Consumo de álcool ( $\geq 175 \mathrm{~g} / \mathrm{sem})$ & $11,3(10,0-12,8)$ \\
\hline Utilização do serviço & Média (DP)/\%(IC) \\
\hline Consultas nos últimos 12 meses & $3,82(5,523)$ \\
\hline Satisfação na última consulta & $84,3(77,5-89,3)$ \\
\hline \multicolumn{2}{|l|}{ Serviço afiliado } \\
\hline ESF & $19,2(9,6-34,7)$ \\
\hline US & $9,9(4,8-19,5)$ \\
\hline CSE Murialdo & $6,2(2,3-15,4)$ \\
\hline SSC-HNSC & $4,4(1,8-10,3)$ \\
\hline CASSI & $6,4(2,2-17,3)$ \\
\hline Ambulatório/PS & $9,9(1,4-46,6)$ \\
\hline Particular & $19,2(3,3-62,7)$ \\
\hline SMET & $2,1(0,4-9,8)$ \\
\hline Clínica Domiciliar & $2,3(0,4-11,1)$ \\
\hline Hospitais e Pronto Atendimento & $20,2(6,9-46,2)$ \\
\hline Médico de referência & $48,1(41,4-54,9)$ \\
\hline Imunização Gripe/pneumococo & $16,1(13,2-19,4)$ \\
\hline Hospitalização em 12 meses & $10,3(8,4-12,5)$ \\
\hline
\end{tabular}

com o desfecho (grau de autopercepção de saúde) foi avaliada no nível hierárquico a que estas pertencem. Uma significância $\leq 0,20$ foi adotada para permanência da variável no modelo multivariado, de acordo como ajuste observado no seu nível hierárquico. As análises estatísticas foram realizadas no programa STATA versão 9, por meio da regressão de Poisson modificada, controlando-se para efeito de clusters do processo de amostragem. Foi adotado nível de significância de $5 \%$ em todas as análises ${ }^{12}$.

\section{Resultados}

Foram entrevistados 3.009 usuários adultos, apresentando idade média de 45,7 anos (DP: 17,3), cor autorreferida predominantemente branca (68,5\%; IC95\%: 61,4-74,8\%) e 59,5\% (IC95\%: 55,8-63\%) eram mulheres. A escolaridade média encontrada foi de 9,5 anos (DP: 4,69). A população apresentou renda per capita média de R \$ 956,20 (DP: $\mathrm{R} \$ 1.286,5)$ e renda familiar média de $\mathrm{R} \$ 2.776,70$ (DP: $\mathrm{R} \$ 3.251,50)$, sendo que classes sociais C, D ou E continham $55,3 \%$ dos entrevistados. As características sociodemográficas e econômicas da população podem ser encontradas na Tabela 1. Observa-se que 30,6\% (IC95\%: 26,8-34,6\%) dos entrevistados estavam empregados (com carteira assinada ou não), 25,2\% (IC95\%: 20-31,1\%) recebiam algum tipo de benefício (aposentadoria, auxílio-doença ou pensionista), e 7,14\% (IC95\%: 5,1-9,9\%) eram desempregados. Saneamento básico, água encanada e coleta adequada de lixo por caminhão estavam presentes em 98,2\% (IC95\%: 97,3$98,8 \%)$ das casas visitadas, sendo essas moradias qualificadas como construídas com segurança em 97,27\% (IC95\%: 95,6-98,3\%) dos casos.

O estado de saúde da população também está apresentado na Tabela 1, abordando alguns aspectos como presença de doenças crônicas e fatores de risco à saúde. Hipertensão atingiu valores de $25 \%$, enquanto diabetes mellitus e cardiopatia isquêmica 7,1 e 5,8\%, respectivamente. Relevante presença de fatores de risco, como sedentarismo $(51,9 \%$; IC95\%: 49,5-54,3\%), tabagismo (47,6\%; IC95\%: 43,1$52,2 \%)$ e consumo de álcool a cima de $175 \mathrm{~g}$ por semana (11,3\%; IC95\%: 10,0-12,8\%) foram encontrados nessa amostra. Ao se observar a utilização do Serviço de Saúde, os usuários realizaram, em média, 3,8 (DP: 5,523) consultas nos últimos 12 meses, sendo que a satisfação na última consulta atingia valores de 84,3\% (IC95\%: 77,5-89,4\%). Cerca de $50 \%$ dos entrevistados possuíam médico de referência, sendo principalmente atendidos por equipes de ESF (19,2\%; IC95\%: 9,6-34,7\%), atendimento particular $(19,2 \%$; IC95\%: 3,3-62,7\%) ou hospitais e pronto atendi- 
mento (20,2\%; IC95\%: 7-46,2\%). Hospitalização nos últimos 12 meses, por qualquer motivo, foi observada em cerca de $10 \%$ dos entrevistados.

Boa autopercepção de saúde foi referida por 2.355 (78,3\%; IC95\%: 74,9-81,3\%), dos quais 1.013 (43\%; IC95\%: 39,3-46,9\%) eram homens, como pode ser observado na Tabela 2. Indivíduos na faixa etária de 18 a 45 anos perceberam a sua saúde melhor do que os com idade superior a 46 anos. As classes sociais C, D e E (52,2\%; IC95\%: 40,2-63,9\%) apresentaram melhor autopercepção de saúde. Em relação aos hábitos de vida, 1.075 indivíduos eram tabagistas (45,7\%; IC95\%: 40,9-50,6\%), 1.197 eram sedentários (45,7\%; IC95\%: 40,9-50,6\%), 276 consomem mais de $175 \mathrm{~g}$ de álcool/semana (11,7\%; IC95\%: 10,2-13,4\%)

Tabela 2: Associação entre variáveis demográficas e socioeconômicas com autopercepção de saúde, Porto Alegre, 2007

\begin{tabular}{|c|c|c|c|}
\hline \multirow{2}{*}{$\begin{array}{l}\text { Variáveis socioeconômicas } \\
\text { e sociodemográficas }\end{array}$} & \multicolumn{3}{|c|}{ Autopercepção de Saúde } \\
\hline & Ruim \%(IC) & Boa \%(IC) & Valor de $p$ \\
\hline Sexo & & & $<0,001$ \\
\hline Masculino & $31,7(27,3-36,4)$ & $43,0(39,3-46,9)$ & \\
\hline Feminino & $68,3(63,6-72,7)$ & $57,0(53,2-60,7)$ & \\
\hline Classe social & & & $<0,001$ \\
\hline$A B$ & $33,6(24,9-43,7)$ & $47,8(36,1-59,8)$ & \\
\hline $\mathrm{CDE}$ & $66,4(56,3-75,2)$ & $52,2(40,2-63,9)$ & \\
\hline Escolaridade (anos completos) & & & $<0,001$ \\
\hline 0 & $5,8(3,9-8,5)$ & $1,9(1,3-2,8)$ & \\
\hline $1-8$ & $49,0(40,6-57,4)$ & $38,8(29,4-49,2)$ & \\
\hline $9-12$ & $29,7(25,2-34,7)$ & $35,2(32,5-38,0)$ & \\
\hline$\geq 13$ anos & $15,5(9,8-23,6)$ & $24,1(14,8-36,7)$ & \\
\hline \multicolumn{4}{|l|}{ Idade estratificada (anos) } \\
\hline $18-30$ & $24,9(19,7-31,0)$ & $26,7(26,5-30,1)$ & 0,02 \\
\hline $31-45$ & $20,5(17,4-24,0)$ & $26,6(21,5-32,5)$ & \\
\hline $46-57$ & $27,8(23,1-33,1)$ & $23,7(21,4-26,3)$ & \\
\hline$\geq 58$ & $26,8(20,2-34,5)$ & $23,0(17,5-29,6)$ & \\
\hline Cor autorreferida & & & 0,18 \\
\hline Branca & $65,39(59,2-71,1)$ & $69,4(61,6-76,2)$ & \\
\hline Não-branca & $34,6(28,9-40,8)$ & $30,6(23,8-38,4)$ & \\
\hline Estado narital & & & 0,05 \\
\hline Casado & $59,1(55,1-62,96)$ & $61,7(59,7-63,6)$ & \\
\hline Solteiro & $20,7(16,9-25,0)$ & $22,5(20,4-24,8)$ & \\
\hline Viúvo/divorciado & $20,2(16,2-24,9)$ & $15,8(14,6-17,1)$ & \\
\hline Renda familiar (R\$) & & & $<0,001$ \\
\hline$>3.200$ & $16,1(9,7-25,7)$ & $27,3(16,2-42,2)$ & \\
\hline $1.601|-| 3.200$ & $19,3(15,0-24,3)$ & $25,7(22,5-29,1)$ & \\
\hline $831|-| 1.600$ & $30,6(25,8-35,8)$ & $24,4(19,0-30,9)$ & \\
\hline $3611-1830$ & $24,0(18,2-31,0)$ & $16,0(11,4-21,8)$ & \\
\hline $0 \mid-1360$ & $10,03(7,2-13,8)$ & $6,6(5,37-8,08)$ & \\
\hline Ocupação & & & $<0,001$ \\
\hline Empregado & $25,3(20,8-30,3)$ & $32,0(28,0-36,4)$ & \\
\hline Autônomo/dono & $11,8(9,4-14,7)$ & $15,0(13,1-17,0)$ & \\
\hline Com benefício & $31,1(24,6-38,4)$ & $2,4(18,3-29,6)$ & \\
\hline Estudante/do lar/outros & $24,0(21,2-27,1)$ & $22,6(20,5-24,7)$ & \\
\hline Desempregado & $7,8(5,4-11,2)$ & $7,0(4,8-9,9)$ & \\
\hline \multicolumn{4}{|l|}{ Saneamento básico } \\
\hline Presença & $97,4(95,2-98,6)$ & $98,4(97,5-99,0)$ & 0,09 \\
\hline Ausência & $2,6(1,4-4,8)$ & $1,6(1,0-2,5)$ & \\
\hline \multicolumn{4}{|l|}{ Moradores por casa } \\
\hline $1 \mid-12$ & $28,1(23,7-33,0)$ & $26,0(20,8-31,9)$ & 0,022 \\
\hline $3 \mid-15$ & $57,8(54,5-61,0)$ & $63,3(59,9-66,5)$ & \\
\hline $6|-| 13$ & $14,1(10,16-19,3)$ & $10,7(7,3-15,5)$ & \\
\hline \multicolumn{4}{|l|}{ Tipo de moradia } \\
\hline Material seguro & $95,9(92,7-97,7)$ & $97,7(96,0-98,6)$ & 0,054 \\
\hline Material não seguro & $4,1(2,3-7,2)$ & $2,3(1,4-4,0)$ & \\
\hline
\end{tabular}

\begin{tabular}{|c|c|c|c|}
\hline Estado de saúde & Ruim \%(IC) & Boa \%(IC) & Valor de $p$ \\
\hline $\begin{array}{l}\text { Imunização (gripe/ } \\
\text { pneumococo) }\end{array}$ & & & 0,2 \\
\hline Não vacinado & $82,2(77,0-86,4)$ & $84,4(81,2-87,1)$ & \\
\hline Vacinado & $17,8(13,6-23,0)$ & $15,6(12,8-18,8)$ & \\
\hline Tabagismo & & & $<0,001$ \\
\hline Não tabagista & $45,5(41,8-49,2)$ & $54,3(49,4-59,1)$ & \\
\hline Tabagista & $54,5(50,8-58,1)$ & $45,7(40,9-50,6)$ & \\
\hline $\begin{array}{l}\text { Consumo de álcool (g/ } \\
\text { semana) }\end{array}$ & & & 0,2 \\
\hline $01-175$ & $90,1(87,4-92,2)$ & $88,3(86,6-89,7)$ & \\
\hline$\geq 175$ & $9,9(7,8-12,6)$ & $11,7(10,3-13,4)$ & \\
\hline Sedentarismo & & & 0,3 \\
\hline Não Sedentário & $46,2(41,6-51,0)$ & $48,6(46,3-50,9)$ & \\
\hline Sedentário & $53,7(49,0-58,4)$ & $51,4(49,1-53,7)$ & \\
\hline Doença crônica & & & $<0,001$ \\
\hline Ausência & $53,36(47,8-58,9)$ & $75,6(71,8-79,1)$ & \\
\hline Presença & $46,6(41,1-52,2)$ & $24,4(20,9-28,2)$ & \\
\hline $\begin{array}{l}\text { Hospitalização nos últimos } \\
12 \text { meses }\end{array}$ & & & $<0,001$ \\
\hline Sim & $15,4(12,6-18,8)$ & $8,84(7,0-11,1)$ & \\
\hline Não & $84,6(81,2-87,4)$ & $91,2(88,9-93,0)$ & \\
\hline Utilização do serviço & Ruim \% (IC) & Boa \% (IC) & Valor de $p$ \\
\hline Serviço afiliado & & & $<0,001$ \\
\hline ESF & $22,5(12,3-37,5)$ & $18,3(8,9-34,0)$ & \\
\hline US & $11,3(6,0-20,2)$ & $9,6(4,4-19,5)$ & \\
\hline CSE Murialdo & $8,1(3,3-18,5)$ & $5,7(2,1-14,6)$ & \\
\hline SSC-HNSC & $6,3(2,8-13,5)$ & $4,0(1,6-9,6)$ & \\
\hline CASSI & $5,2(1,7-14,6)$ & $6,7(2,3-18,2)$ & \\
\hline Ambulatório/PS & $9,0(1,3-43,3)$ & $10,1(1,4-47,6)$ & \\
\hline Particular & $13,6(2,1-53,0)$ & $20,8(3,6-64,8)$ & \\
\hline $\begin{array}{l}\text { Serviço Médico no } \\
\text { Trabalho }\end{array}$ & $1,7(0,3-8,3)$ & $21,7(0,4-10,2)$ & \\
\hline Clínica Domiciliar & $1,7(0,3-8,3)$ & $2,4(0,4-11,9)$ & \\
\hline $\begin{array}{l}\text { Hospitais e Pronto } \\
\text { Atendimento }\end{array}$ & $20,6(7,3-46,2)$ & $20,1(6,8-46,4) 1$ & \\
\hline Médico de referência & & & 0,006 \\
\hline Possui & $52,0(45,1-58,9)$ & $47,0(40,2-54,0)$ & \\
\hline Não possui & $48,0(4,1-54,9)$ & $53,0(46,0-59,8)$ & \\
\hline $\begin{array}{l}\text { Consultas nos últimos } 12 \\
\text { meses }\end{array}$ & & & $<0,001$ \\
\hline$<4$ & $63,7(58,4-68,6)$ & $77,2(71,8-81,9)$ & \\
\hline$\geq 4$ & $36,3(31,3-41,5)$ & $22,8(18,1-28,2)$ & \\
\hline $\begin{array}{l}\text { Satisfação na última } \\
\text { consulta }\end{array}$ & & & $<0,001$ \\
\hline Não satisfeito & $22,8(17,5-29,1)$ & $13,6(8,8-20,4)$ & \\
\hline Satisfeito & 7,7 (70,8-82,5) & $86,4(79,6-91,2)$ & \\
\hline
\end{tabular}

ESF: Estratégia Saúde da Família; US: Unidade de Saúde; CSE Murialdo: Centro de Saúde Escola Murialdo; SSC-HNSC: Serviço de Saúde Comunitária do Hospital Nossa Senhora da Conceição ; CASSI: Caixa de Assistência dos Funcionários do Banco do Brasil. 
Tabela 3: Variáveis associadas a boa autopercepção de saúde entre os usuários da rede de Atenção Primária à Saúde, Porto Alegre, 2007

\begin{tabular}{|c|c|c|c|c|c|}
\hline \multirow[t]{2}{*}{ Socioeconômicas } & \multicolumn{2}{|c|}{$\begin{array}{c}\text { Modelo de Poisson } \\
\text { Univariado }\end{array}$} & \multicolumn{3}{|c|}{$\begin{array}{l}\text { Modelo de Poisson } \\
\text { Multivariado }\end{array}$} \\
\hline & $\mathrm{RP}$ & Valor de $p$ & $\mathrm{RP}$ & IC (95\%) & Valor de $p$ \\
\hline \multicolumn{6}{|l|}{ Classe social } \\
\hline CDE & 1 & - & 1 & - & - \\
\hline $\mathrm{AB}$ & 1,13 & 0,000 & 1,01 & $0,97-1,06$ & 0,470 \\
\hline \multicolumn{6}{|l|}{ Renda familiar ( $\mathrm{R} \$$ ) } \\
\hline$>3.200$ & 1 & - & 1 & - & - \\
\hline $1.601|-| 3.200$ & 0,96 & 0,154 & 0,93 & $0,82-1,06$ & 0,277 \\
\hline $831|-| 1.600$ & 0,86 & $<0,001$ & 0,98 & $0,89-1,08$ & 0,708 \\
\hline $361|-| 830$ & 0,82 & $<0,001$ & 1,07 & $0,94-1,20$ & 0,274 \\
\hline $0|-| 360$ & 0,82 & $<0,001$ & 1,09 & 0,99-120 & 0,069 \\
\hline \multicolumn{6}{|l|}{$\begin{array}{l}\text { Escolaridade } \\
\text { (anos completos) }\end{array}$} \\
\hline 0 & 1 & - & 1 & - & - \\
\hline 1-8 anos & 1,38 & $<0,001$ & 1,24 & $1,03-1,50$ & 0,021 \\
\hline 9-12 anos & 1,51 & $<0,001$ & 1,28 & $1,06-1,53$ & 0,008 \\
\hline$\geq 13$ anos & 1,58 & $<0,001$ & 1,26 & $1,04-1,52$ & 0,014 \\
\hline \multicolumn{6}{|l|}{ Idade (anos) } \\
\hline $18-30$ & 1 & - & 1 & - & - \\
\hline $31-45$ & 1,03 & 0,145 & 1,04 & $0,99-1,10$ & 0,086 \\
\hline $46-57$ & 0,95 & 0,186 & 1,01 & $0,92-1,05$ & 0,551 \\
\hline$\geq 58$ & 0,95 & 0,065 & 1,03 & $0,97-1,08$ & 0,359 \\
\hline \multicolumn{6}{|l|}{ Sexo } \\
\hline Masculino & 1 & - & 1 & - & - \\
\hline Feminino & 0,90 & $<0,001$ & 0,94 & $0,91-0,97$ & $<0,001$ \\
\hline \multicolumn{6}{|l|}{ Tipo de moradia } \\
\hline Material seguro & 1 & - & 1 & - & - \\
\hline Material não-seguro & 0,85 & 0,102 & 0,91 & $0,74-1,12$ & 0,381 \\
\hline \multirow[t]{2}{*}{ Estado de saúde } & \multicolumn{2}{|c|}{$\begin{array}{c}\text { Modelo de Poisson } \\
\text { Univariado }\end{array}$} & \multicolumn{3}{|c|}{$\begin{array}{l}\text { Modelo de Poisson } \\
\text { Multivariado }\end{array}$} \\
\hline & $\mathrm{RP}$ & Valor de $p$ & $\mathrm{RP}$ & IC (95\%) & Valor de $p$ \\
\hline \multicolumn{6}{|l|}{ Tabagismo } \\
\hline Não tabagista & 1 & - & 1 & - & - \\
\hline Tabagista & 0,92 & $<0,001$ & 0,96 & $0,92-1,00$ & 0,059 \\
\hline \multicolumn{6}{|l|}{$\begin{array}{l}\text { Consumo de álcool (g/ } \\
\text { semana) }\end{array}$} \\
\hline $0 \mid-175$ & 1 & - & - & - & - \\
\hline$\geq 175$ & 0,98 & 0,351 & - & - & - \\
\hline \multicolumn{6}{|l|}{ Doenças crônicas } \\
\hline Ausência & 1 & - & 1 & - & - \\
\hline Presença & 0,78 & $<0,001$ & 0,81 & $0,76-0,87$ & $<0,001$ \\
\hline \multicolumn{6}{|c|}{$\begin{array}{l}\text { Hospitalização nos últimos } \\
12 \text { meses }\end{array}$} \\
\hline Sim & 1 & - & 1 & - & - \\
\hline Não & 1,18 & $<0,001$ & 1,09 & $1,01-1,19$ & 0,040 \\
\hline \multirow[t]{2}{*}{ Utilização do Serviço } & \multicolumn{2}{|c|}{ Modelo de Poisson } & \multicolumn{3}{|c|}{ Modelo de Poisson } \\
\hline & $\mathrm{RP}$ & Valor de $p$ & $\mathrm{RP}$ & IC (95\%) & Valor de $p$ \\
\hline
\end{tabular}

\begin{tabular}{|c|c|c|c|c|c|}
\hline \multicolumn{6}{|c|}{$\begin{array}{l}\text { Consultas nos últimos } 12 \\
\text { meses }\end{array}$} \\
\hline$<4$ & 1 & - & 1 & - & - \\
\hline$\geq 4$ & 0,86 & $<0,001$ & 0,89 & $0,85-0,93$ & $<0,001$ \\
\hline \multicolumn{6}{|c|}{$\begin{array}{l}\text { Satisfação na última } \\
\text { consulta }\end{array}$} \\
\hline Não satisfeito & 1 & - & 1 & - & - \\
\hline Satisfeito & 1,19 & $<0,001$ & 1,17 & $1,10-1,24$ & $<0,001$ \\
\hline
\end{tabular}

e 573 possuem pelo menos uma doença $(24,4 \%$; IC95\%: 20,9-28,2\%); todos esses perceberam sua saúde como boa (Tabela 2). Em moradias com três a cinco habitantes, encontrou-se a maior porcentagem de boa autoperceção $(63,3 \%$; IC95\%: 59,9-66,5\%) bem como em famílias cuja renda era superior a R $\$ 3.200,00$ por mês $(27,3 \%$; IC95\%: $16,2-$ $42,2 \%)$ e entre indivíduos que estavam empregados (32\%; IC95\%: 28-36,4\%) (Tabela 2).

Pelo modelo hierárquico univariado, observa-se que variáveis dos diferentes níveis, como sexo feminino (RP: 0,92; IC95\%: 0,88-0,95), tabagismo (RP: 0,92; IC95\%: 0,88-0,96), presença de doença crônica (RP: 0,78; IC95\%: 0,72-0,84) e utilização do Serviço - pelo menos quatro consultas/ano (RP: 0,85; IC95\%: 0,82-0,92), são fatores que diminuem a probabilidade de uma pessoa referir boa autopercepção de saúde. Não ter hospitalização no último ano (RP: 1,14; IC95\%: 1,06-1,25) e estar satisfeito com a última consulta (RP: 1,19; IC95\%: 1,11-1,27) aumentaram significativamente a probabilidade de o usuário avaliar sua saúde como boa (Tabela 3).

Ajustando um modelo multivariado com essas variáveis, identifica-se que algumas delas perdem a significância estatística, mas a relação com a boa autopercepção em saúde se mantém na mesma direção. Nessa análise, as variáveis "ter hospitalização no último ano" (RP: 1,09; IC95\%: 1,01-1,19), "satisfação com a última consulta" (RP: 1,17; IC95\%: 1,10-1,24) e escolaridade (1 a 8 anos, RP: 1,24; IC95\%: 1,03-1,50; 9 a 12 anos, RP: 1,28; IC95\%: 1,061,53 ; $\geq 13$ anos, RP: 1,26; IC95\%: 1,04-1,52) foram as mais significativas (Tabela 3 ).

\section{Discussão}

Neste estudo, a autopercepção de saúde foi considerada boa pela maioria dos indivíduos, semelhante ao que foi encontrado para Porto Alegre em estudo realizado pelo Instituto Nacional do Câncer (INCA) em 2002-20039. Entretanto, neste estudo, não ocorreu associação significativa entre a ausência de doenças crônicas e fatores de risco à saúde, que em outros estudos apresentavam associação independente ${ }^{9,16}$.

Fatores socioeconômicos, como escolaridade, classe social e renda, atuaram de maneira significativa na descrição da autopercepção da saúde. Em indivíduos com escolaridade mais elevada (acima de 13 anos), a boa autopercepção de saúde foi menos referida $(18 \%)$ em comparação àqueles de menor escolaridade (1 a 8 anos) que apresentaram $30 \%$ de boa percepção de saúde. Esse fato se repetiu quando foi avaliada a amostra por classe social, pois classes sociais A e B referiram ter boa 
autopercepção de saúde em menor proporção do que as classes C, D e E. Isso poderia ser atribuído a uma maior expectativa do significado de boa saúde para as classes sociais mais altas, influenciada diretamente pela menor prevalência e incidência de doença nesses segmentos sociais ${ }^{3,17-19}$.

Os indivíduos em faixas etárias mais elevadas referiram pior autopercepção de saúde do que os mais novos, assim como em outros estudos ${ }^{20,21}$. Segovia et al. ${ }^{22}$ mostraram que a percepção do nível de saúde está associada à adoção de comportamentos promotores de saúde, e as evidências indicam que a prevalência de comportamentos de risco é maior entre sujeitos com percepção negativa de saúde, isto é, aqueles que consideram sua saúde atual como regular ou ruim ${ }^{22}$. Neste estudo, observamos que indivíduos com fatores de risco à saúde como tabagismo e sedentarismo, além da presença de doenças crônicas já estabelecidas, consideravam sua saúde de maneira positiva, evidenciando uma variação entre o estado real de saúde do paciente e sua autopercepção.

Essa avaliação contemplou apenas a experiência dos usuários e de forma quantitativa, não abordando questões que podem ser avaliadas qualitativamente. Por se tratar de um estudo transversal, os resultados aqui discutidos não estão livres da causalidade reversa. Quando define-se no modelo hierárquico "doença crônica" como determinante da autopercepção de saúde, não se há garantia de que essa relação seja inversa, isto é, que ter boa autopercepção de saúde leve à ausência de doenças crônicas.

\section{Conclusões}

A utilização da autopercepção de saúde para explicar o processo saúde-doença depende de suas características subjetivas, nas quais também se inserem suas limitações. Esta também pode ser influenciada pela cultura, expectativas, visão de mundo e pela personalidade dos indivíduos, entretanto, por ser de fácil aplicação, pode ser usada tanto em pesquisa, como na prática clínico-assistencial, em diferentes momento para acompanhar a evolução da saúde dos pacientes.

Neste estudo, a boa autopercepção da saúde mostra-se influenciada por fatores biológicos, socioeconômicos e de vínculo com o Serviço. Cabe ressaltar que maior satisfação com Serviço de APS estava associada à melhor autopercepção de saúde, justificando a reorganização do Sistema Único de Saúde (SUS) por meio da ampliação da rede de serviços de APS, como a ESF.

\section{Referências}

1. Starfield B. Atenção primária: equilíbrio entre necessidade de saúde, serviços e tecnologia. Brasília: UNESCO; 2002. 726p.

2. Brasil. Ministério da Saúde. Secretaria de Atenção Básica. Departamento de Atenção Básica. Política Nacional de Atenção Básica. Brasília, DF; 2006. 60p. Série Pactos pela Saúde. Volume 4.

3. Silva NN, Pedroso GC, Puccini RF, Furlani WJ. Desigualdades sociais e uso de serviços de saúde: evidências de análise estratificada. Rev Saúde Pública. 2000;34(1):44-9.

4. Macinko J, Guanais FC, Souza MFM. Evaluation of the impact of the Family Health Program on infant mortality in Brazil, 1990-2002. J Epidemiol Community Health. 2006;60:13-9.

5. Oliveira IBN. Acesso universal? Obstáculos ao acesso, continuidade do uso e gênero em um serviço especializado em HIV/AIDS em Salvador, Bahia, Brasil. Cad Saúde Pública. 2009;25(Supl.2):s259-s268.

6. Dias-da-Costa JS, Olinto MTA, Bassani D, Marchionatti CRE, Bairros FS, Oliveira MLP, et al. Desigualdades na realização do exame clínico de mama em São Leopoldo, Rio Grande do Sul, Brasil. Cad Saúde Pública 2007;23(7):1603-12.

7. de Bruin A, Picavet HS, Nossikov A. Health interview surveys: Towards International Harmonization of Methods and Instruments. WHO Reg Publ Eur Ser. 1996;58:i-161.

8. Jylhä M, Guralnik JM, Ferrucci L, Jokela J, Heikkinen E. Is self-rated health comparable across cultures and genders? J Gerontol B Psychol Sci Soc Sci. 1998;53(3):S144-52.

9. Lima-Costa MF, Firmo JO, Uchôa E. [The structure of self-rated health among older adults: the Bambuí health and ageing study (BHAS)]. Rev Saude Publica. 2004;38(6):827-34.

10. Theme Filha MM, Szwarcwald CL, Souza Junior PR. [Measurements of reported morbidity and interrelationships with health dimensions]. Rev Saude Publica. 2008;42 (1):73-81.

11. Segovia J, Bartlett RF, Edwards AC. An empirical analysis of the dimensions of health status measures. Soc Sci Med. 1989;29(6):761-8.

12. Harzheim E, Duncan BB, Stein AT, Cunha CRH, Gonçalves MR, Trindade $R G$, et al [Internet]. Quality and effectiveness of different approaches to primary care delivery in Brazil. BMC Health Services Research. 2006. (Cited 2010 Jul. 27). Available from: http://www.biomedcentral. com/1472-6963/6/156. DOI: 6:156doi:10.1186/1472-6963-6-156.

13. Shi L, Starfield B, Jihaong $X$. Validating the adult primary care assessment tool. J Family Practice. 2001:50:161-75.

14. Kloetzel K, Bertoni AM, Irazoqui MC, Campos VP, Santos RN. [Quality control in primary health care. I - Consumer satisfaction]. Cad Saude Publica. 1998;14:623-8.

15. Corrao G, Bagnardi V, Zambon A, Arico S. Exploring the dose-response relationship between alcohol consumption and the risk of several alcoholrelated conditions: a meta-analysis. Addiction. 1999;94(10):1551-73.

16. Szwarcwald CL, Bastos FI, Barcellos C, Pina MF, Esteves MA. Health conditions and residential concentration of poverty: a study in Rio de Janeiro, Brazil. J Epidemiol Community Health. 2000;54(7):530-6.

17. Blumenthal D, Mort E, Edwards J. The efficacy of primary care for vulnerable population groups. Health Serv Res. 1995;30:253-73.

18. Shi L, Starfield B, Politzer R, Regan J. Primary care, self-rated health, and reductions in social disparities in health. Health Serv Res. 2002;37:52950.

19. Ma J, Stafford RS. Quality of US outpatient care: temporal changes and racial/ethnic disparities. Arch Intern Med. 2005;165:1354-61.

20. Lima-Costa MF, Firmo JO, Uchôa E. Differences in self-rated health among older adults according to socioeconomic circumstances: the Bambuí Health and Aging Study. Cad Saude Publica. 2005;21(3):830-9.

21. Wong R, Pelaez M, Palloni A. [Self-reported general health in older adults in Latin America and the Caribbean: usefulness of the indicator]. Rev Panam Salud Publica. 2005;17(5-6):323-32.

22. Segovia J, Bartlett RF, Edwards AC. The association between selfassessed health status and individual health practices. Can $\mathrm{J}$ Public Health. 1989;80(1):32-7. 